\title{
基于索驱动的大型柔性结构振动抑制策略研究“
}

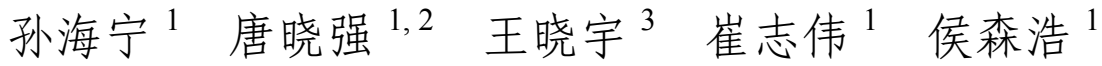 \\ (1. 清华大学机械工程系 北京 100084;
}

2. 清华大学精密超精密制造装备及控制北京市重点实验室 北京 100084;

3. 北京空间飞行器总体设计部 北京 100094)

\begin{abstract}
摘要: 柔性索并联机构具有工作空间大、能耗低和惯量小等优点, 适用于长距离柔性析架结构的振动主动抑制。首先提出一 种四索驱动的柔性体抑振并联机构, 根据闭环矢量原理和机构的几何特征, 对四索并联机构进行运动学分析。接着建立四索 并联机构的静力学模型, 并将超长尺度柔性析架机构的往复振动简化为梁的弯曲振动, 利用振型叠加法求解外部激励作用下 的强迫响应。最后针对超长尺度柔性桁架机构的振动抑制问题, 提出一种基于模糊控制与 PID 控制相结合的控制策略, 通过 仿真验证所提控制策略的有效性。结果表明, 所设计的柔索并联抑振系统具有明显的抑振效果, 柔性析架的振幅与振动衰减 时间大幅降低, 且系统的四索索力值能够在合适范围内变化。
\end{abstract}

关键词: 索并联机构; 大型柔性结构；振动抑制；模糊 PID 控制

中图分类号: TP242

\section{Vibration Suppression of Large Flexible Structure Based on Cable-driven Parallel Robots}

\section{SUN Haining $^{1} \quad$ TANG Xiaoqiang $^{1,2} \quad$ WANG Xiaoyu $^{3} \quad$ CUI Zhiwei ${ }^{1}$ HOU Senhao $^{1}$}

(1. Institute of Manufacturing Engineering, Tsinghua University, Beijing 100084;

2. Beijing Key Lab. of Precision/Ultra-Precision Manufacturing Equipment and Control, Tsinghua University, Beijing 100084;

3. Beijing Institute of Spacecraft System Engineering, Beijing 100094)

\begin{abstract}
With the benefits of large workspace, low energy consumption and small inertia, a flexible cable-driven parallel robot (CDPR) is suitable for vibration suppression of long-size flexible truss mechanism. A flexible cable-driven parallel mechanism used for vibration attenuation of large-size flexible truss is presented. Kinematics and static model of the CDPR is analyzed by principle of closed loop vector and geometric characteristic of mechanism. The long-size flexible truss is simplified as a beam, and the mode superposition method is used to solve the forced response under external excitation. Vibration suppression of the long-size flexible structure with model uncertainties are considered. A control strategy based on fuzzy and PID control scheme is proposed. The simulation results show that the presented system have great affection on the vibration suppression of the long-size flexible truss, whose amplitude and vibration decay time are significantly reduced. In addition, four cable tension values can be changed within a suitable range.
\end{abstract}

Key words: cable parallel manipulator; large flexible truss mechanism; vibration attenuation; fuzzy PID control

\section{0 前言}

随着我国航天技术的发展, 各种各样的卫星如 侦察卫星、气象卫星和通讯卫星等逐渐成为空间环

* 国家自然科学基金资助项目(91648107)。20180613 收到初稿, 20181228 收到修改稿
境探测的主体，大型柔性结构件也因此得到了广泛 应用。针对特殊用途, 有的卫星单侧展翼长度可达 $100 \mathrm{~m}$ ，广泛采用各种柔性桁架结构支撑有效载荷。 携带柔性伸杆机构的航天器工作于真空、微重力状 态, 受外部扰动或本体机动, 极易产生振动, 严重 影响其指向精度和空间姿态稳定性 ${ }^{[1]}$ 。因此, 超长 尺度柔性桁架结构的振动抑制问题是这类航天器设 计的关键技术之一。 
近年来, 有许多控制方法应用于卫星柔性展翼 的主动振动抑制, 如自适应滑膜控制 ${ }^{[2]}$, 自适应超 扭曲 ${ }^{[3]}$ 和鲁棒控制 ${ }^{[4]}$ 等方法。 JI 等 ${ }^{[5]}$ 将卫星对称的展 翼等效成为欧拉伯努利梁, 并用偏微分方程表示, 通过反向递推法建立抑制振动的控制策略, 并利用 双曲函数与 Nussbaum 函数建立辅助系统以满足输 入的约束条件。陶佳伟等 ${ }^{[6]}$ 针对挠性航天器姿态机 动过程中存在模型参数难以确定、外界干扰、执行 机构饱和受限、挠性附件振动及挠性模态不易直接 测量等问题, 提出了一种鲁棒自适应控制方法, 来 处理系统中的不确定参数和外界干扰上界。所有这 些研究均取得了较好的控制效果, 然而关于空间桁 架结构振动的研究还很少。许建国等 ${ }^{[7]}$ 采用压电杆 件进行优化配置实现桁架结构的振动主动控制, 最 优配置下控制电压的峰值在 $400 \mathrm{~V}$ 左右, 并采用最 优模态控制算法进行振动抑制。张金龙等 ${ }^{[8]}$ 提出了 一种在驱动机构与太阳翼之间安装旋转磁流变阻尼 器的系统振动抑制方案, 通过控制旋转运动实现展 翼的振动抑制, 但对于长度达百米的柔性桁架结构 却不适用。

从 20 世纪 80 年代开始, 柔索并联机构引起了 研究人员的兴趣, 并广泛应用于起重和装配等 ${ }^{[9-10]}$ 。 研究发现, 索驱动并联机构具有以下特点 ${ }^{[11-14]}$ 。

（1）结构简单，用轻质绳索代替传统的刚性构 件，从而具有低能耗和小惯量等的特点。

(2) 绳索只能承受拉力, 不能承受压力, 且受 力方向只能沿着绳索。

(3) 工作空间大。

基于上述柔索并联机构的特点, 本文将提出一 种新的基于索驱动并联机构的超长尺度桁架结构振 动抑制的方案, 用于卫星展翼的振动抑制, 具体如 图 1 所示。分别由 4 根绳索控制卫星的一侧展翼, 通过选取桁架上最佳较接点作为绳索的附着点, 绳 索另一端经由滚筒与伺服电动机系统连接, 通过控 制绳索的张紧来改变绳索对桁架结构的拉力, 建立 模糊 PID 控制的抑振策略, 实现桁架结构的振动抑 制。最后，完成证明控制策略有效性的仿真验证。



图 1 柔索并联抑振机构

\section{1 柔索并联抑振机构模型构建}

\section{1 索并联机构运动学建模}

根据设计结构图 1, 卫星单侧展翼由 4 根索驱 动控制, 伺服电动机系统位于卫星本体内部, 其等 效运动模型如图 2 所示。 4 个绳索出索点分布于长 度与宽度分别为 $b$ 和 $a$ 的矩形四个顶点，柔性桁架 杆的铰接点与固定端的水平距离为 $c$, 结点 $B_{1}$ 与 $B_{2}$ 水平距离为 $d$ 。

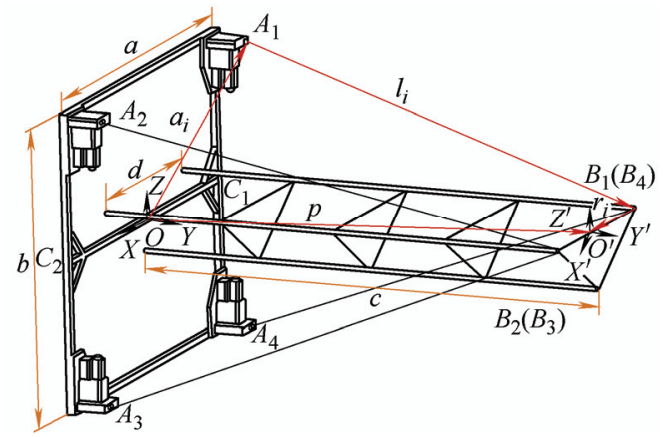

图 2 运动学模型

由于本文研究的索机构应用于太空微重力环 境, 可以忽略绳索的垂度, 用直线模型代替绳索悬 链模型, 所得到的运动学模型如图 2 所示。其中, $O X Y Z$ 为全局坐标系, 原点 $O$ 位于横杆 $C_{1} C_{2}$ 的几何 中心, $Y$ 轴垂直于矩形 $A_{1} A_{2} A_{3} A_{4}$ 所在平面, $Z$ 轴与 矩形 $A_{1} A_{4}$ 边平行, $X$ 轴方向由右手定理决定。 $O^{\prime} X^{\prime} Y^{\prime} Z^{\prime}$ 为局部坐标系, 原点 $O^{\prime}$ 位于结点 $B_{1} B_{2}$ 中 点。 $\boldsymbol{p}$ 为两个坐标系原点 $O O^{\prime}$ 之间平移矢量, 矢量 $\boldsymbol{r}_{i}$ 由桁架铰接点指向原点 $O^{\prime}$, 矢量 $\boldsymbol{l}_{i}$ 为各索矢量。 4 根绳索的方向矢量为

$$
\boldsymbol{l}_{i}=\boldsymbol{p}-\boldsymbol{a}_{i}-R \bullet \boldsymbol{r}_{i}
$$

$$
R=\left(\begin{array}{ccc}
\mathrm{c} \phi \mathrm{c} \gamma & -\mathrm{c} \varphi \mathrm{s} \gamma & \mathrm{s} \phi \\
\mathrm{s} \varphi \mathrm{s} \phi \mathrm{c} \gamma+\mathrm{c} \varphi \mathrm{s} \gamma & -\mathrm{s} \varphi \mathrm{s} \phi \mathrm{s} \gamma+\mathrm{c} \varphi \mathrm{s} \gamma & -\mathrm{s} \varphi \mathrm{c} \phi \\
-\mathrm{c} \varphi \mathrm{s} \phi \mathrm{c} \gamma+\mathrm{s} \varphi \mathrm{s} \gamma & \mathrm{c} \varphi \mathrm{s} \phi \mathrm{s} \gamma+\mathrm{s} \varphi \mathrm{c} \gamma & \mathrm{c} \varphi \mathrm{c} \phi
\end{array}\right)
$$

式中， $\mathrm{c}$ 表示 $\cos , \mathrm{s}$ 表示 $\sin$, 单位索矢量为

$$
\begin{gathered}
\boldsymbol{u}_{i}=\frac{O O^{\prime}-O A_{i}-R \cdot B_{i} O^{\prime}}{\left\|O O^{\prime}-O A_{i}-R \cdot B_{i} O^{\prime}\right\|}=\frac{\boldsymbol{p}-\boldsymbol{a}_{i}-R \bullet \boldsymbol{r}_{i}}{\left\|\boldsymbol{p}-\boldsymbol{a}_{i}-R \bullet \boldsymbol{r}_{i}\right\|} \\
i=1,2,3,4
\end{gathered}
$$

\section{2 索并联机构静力学建模}

对上述四索并联机构受力分析, 得到受力分析 图如图 3 所示。图 3 中 $\boldsymbol{u}_{i}$ 表示第 $i$ 根绳索索力的单 位方向矢量, 利用截面法分析, 选取过桁架与绳索 的铰接点左侧并无限接近于铰接点且垂直于 $Y$ 轴的 截面, $\boldsymbol{F}^{\prime}$ 与 $\boldsymbol{M}^{\prime}$ 分别表示截面左侧桁架内力对截面 
右侧的拉压力与扭矩, 考虑末端加速度的影响, 设 惯性力为 $\boldsymbol{F}_{m}$ 。

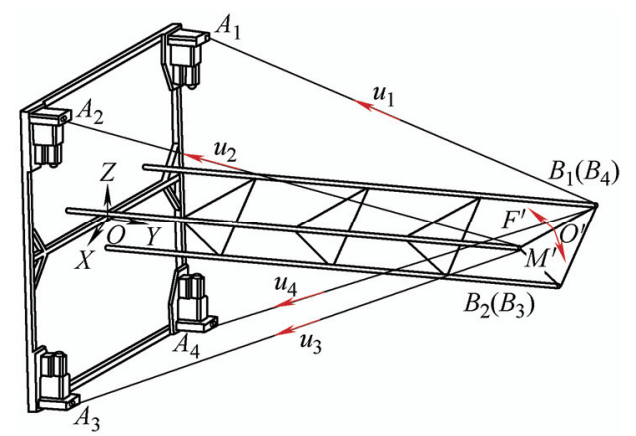

图 3 受力分析图

根据静力平衡条件得到

$$
\begin{gathered}
\left(\begin{array}{ccc}
\boldsymbol{u}_{1 x} & \ldots & \boldsymbol{u}_{4 x} \\
\boldsymbol{u}_{1 z} & \ldots & \boldsymbol{u}_{4 z} \\
\left(\boldsymbol{r}_{1} \times \boldsymbol{u}_{1}\right)_{y} & \ldots & \left(\boldsymbol{r}_{4} \times \boldsymbol{u}_{4}\right)_{y} \\
\left(\boldsymbol{r}_{1} \times \boldsymbol{u}_{1}\right)_{z} & \cdots & \left(\boldsymbol{r}_{4} \times \boldsymbol{u}_{4}\right)_{z}
\end{array}\right)\left(\begin{array}{c}
t_{1} \\
t_{2} \\
t_{3} \\
t_{4}
\end{array}\right)+\left(\begin{array}{c}
\boldsymbol{F}^{\prime}+\boldsymbol{F}_{m} \\
\boldsymbol{M}^{\prime}
\end{array}\right)=0 \\
t_{i}>0 \quad i=1,2,3,4
\end{gathered}
$$

$\left(\begin{array}{cccc}\boldsymbol{u}_{1 x} & \boldsymbol{u}_{2 x} & \boldsymbol{u}_{3 x} & \boldsymbol{u}_{4 x} \\ \boldsymbol{u}_{1 z} & \boldsymbol{u}_{2 z} & \boldsymbol{u}_{3 z} & \boldsymbol{u}_{4 z} \\ \left(\boldsymbol{r}_{1} \times \boldsymbol{u}_{1}\right)_{y} & \left(\boldsymbol{r}_{2} \times \boldsymbol{u}_{2}\right)_{y} & \left(\boldsymbol{r}_{3} \times \boldsymbol{u}_{3}\right)_{y} & \left(\boldsymbol{r}_{4} \times \boldsymbol{u}_{4}\right)_{y} \\ \left(\boldsymbol{r}_{1} \times \boldsymbol{u}_{1}\right)_{z} & \left(\boldsymbol{r}_{2} \times \boldsymbol{u}_{2}\right)_{z} & \left(\boldsymbol{r}_{3} \times \boldsymbol{u}_{3}\right)_{z} & \left(\boldsymbol{r}_{4} \times \boldsymbol{u}_{4}\right)_{z}\end{array}\right) \in \mathbf{R}^{4 \times 4}$

式中, $t_{i}$ 是第 $i$ 根索的索力值, $\boldsymbol{u}_{i x}$ 与 $\boldsymbol{u}_{i z}$ 是第 $i$ 根索 索力的单位方向矢量在 $X$ 轴与 $Y$ 轴的分量, $\left(\boldsymbol{r}_{i} \times \boldsymbol{u}_{i}\right)_{z}$ 与 $\left(\boldsymbol{r}_{i} \times \boldsymbol{u}_{i}\right)_{z}$ 是第 $i$ 根索索力对 $O^{\prime}$ 的力矩在 $Y$ 轴与 $Z$ 轴的分量。

\section{3 柔性梁运动方程}

考虑到的桁架结构的长度可达 $100 \mathrm{~m}$, 远大于 横截面尺寸, 可将其等效为细长梁; 且其振动频率 很低, 可以忽略剪切变形以及截面绕中性轴转动惯 量的影响, 可将其运动简化为在二维平面内欧拉伯 努利梁的振动, 如图 4 所示, 取微段 $\mathrm{d} x$, 如图 5 所 示。以 $y(x, t)$ 表示梁的横向位移, $f(x, t)$ 表示作用 于梁上的单位长度的横向力, 梁的单位体积质量为 $\rho(x)$, 横截面积为 $A(x)$, 弹性模量为 $E$, 横截面 对垂直于 $x$ 和 $y$ 轴且通过横截面形心的轴的惯性矩

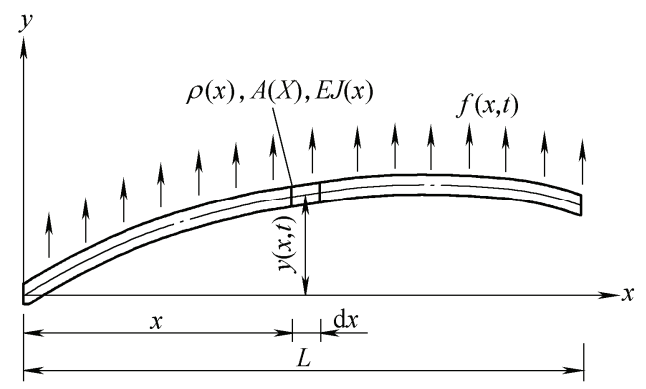

图 4 欧拉-伯努利梁
为 $J(x)$, 对微段进行受力分析得到梁横向振动偏微 分方程 ${ }^{[15]}$ 。

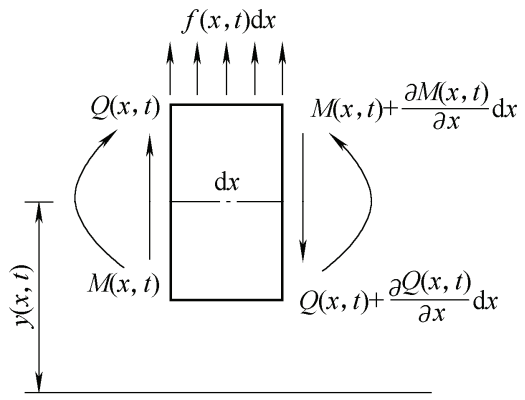

图 5 微段受力分析图

坚直 $y$ 方向上的运动方程

$$
\begin{gathered}
\rho(x) A(x) \mathrm{d} x \frac{\partial^{2} y(x, t)}{\partial t^{2}}= \\
Q-\left(Q+\frac{\partial Q}{\partial x} \mathrm{~d} x\right)+f(x, t) \mathrm{d} x
\end{gathered}
$$

两边同除以 $\mathrm{d} x$, 可以简化为

$$
\rho(x) A(x) \mathrm{d} x \frac{\partial^{2} y(x, t)}{\partial t^{2}}+\frac{\partial Q}{\partial x}=f(x, t)
$$

不计转动惯量和剪切变形的影响, 忽略截面绕 中心轴的转动影响, 微段的转动方程为

$$
\begin{gathered}
M+\frac{\partial M}{\partial x} \mathrm{~d} x-M-Q \mathrm{~d} x- \\
\frac{\partial Q}{\partial x} \mathrm{~d} x \mathrm{~d} x+f(x, t) \mathrm{d} x \frac{\mathrm{d} x}{2}=0
\end{gathered}
$$

略去包含 $d x$ 的二次项，式(5)可以简化为

$$
Q=\frac{\partial M}{\partial x}
$$

代入式(4)可得

$$
\rho(x) A(x) \mathrm{d} x \frac{\partial^{2} y(x, t)}{\partial t^{2}}+\frac{\partial^{2} M}{\partial x^{2}}=f(x, t)
$$

由材料学知，弯矩和挠度有如下关系式

$$
M(x, t)=E J(x) \frac{\partial^{2} y(x, t)}{\partial x^{2}}
$$

将式(8)代入式(7)得梁横向振动的偏微分方程

$$
\begin{gathered}
\rho(x) A(x) \mathrm{d} x \frac{\partial^{2} y(x, t)}{\partial t^{2}}+ \\
\frac{\partial^{2}}{\partial x^{2}}\left[E J(x) \frac{\partial^{2} y(x, t)}{\partial x^{2}}\right]=f(x, t)
\end{gathered}
$$

设在给定边界条件下的固有频率为 $\omega_{i}$, 相应的 振型函数为 $Y_{i}(x)$, 广义坐标为 $q_{i}(t)$, 根据振型叠加 法可得

$$
y(x, t)=\sum_{i=1}^{\infty} Y_{i}(x) q_{i}(t)
$$


将式(11)代入式(10)中可得

$$
\begin{gathered}
\sum_{i=0}^{\infty} \rho(x) A(x) Y_{i}(x) \ddot{q}_{i}(t)+ \\
\sum_{i=0}^{\infty} \frac{\mathrm{d}^{2}}{\mathrm{~d} x^{2}}\left[E J(x) \frac{\mathrm{d}^{2} Y_{i}(x)}{\mathrm{d} x^{2}} q_{i}(t)\right]=f(x, t)
\end{gathered}
$$

式(12)两边同乘以 $Y_{s}(x),(s=1,2, \cdots)$, 在整个长度区 间 $(0<x<L)$ 内积分, 由正则振型函数 $Y_{i}(x)$ 与 $Y_{s}(x)$ 的正交条件可得独立的常微分方程组为

$$
\ddot{q}_{i}(t)+\omega_{i}^{2} q_{i}(t)=Q_{i}(t) \quad i=1,2, \cdots
$$

式中, $q_{i}(t)$ 为对应于广义坐标 $q_{i}(t)$ 的广义力

$$
Q_{i}(t)=\int_{0}^{L} f(x, t) Y_{i}(x) \mathrm{d} x \quad i=1,2, \cdots
$$

考虑模态阻尼方程式(13)可写成

$$
\ddot{q}_{i}(t)+2 \omega_{i} \xi_{i} \dot{q}_{i}(t)+\omega_{i}^{2} q_{i}(t)=Q_{i}(t) \quad i=1,2, \cdots
$$

式中, $\xi_{i}$ 是模态阻尼比。

考虑绳索拉力对柔性梁作用力在坚直方向的分 力, 且设作用点横坐标为 $x_{f}$, 则

$$
f(x, t)=f(t) \delta\left(x-x_{f}\right)
$$

仅考虑柔性梁横向振动的前 $N$ 阶模态, 可得矩 阵方程

$$
\begin{gathered}
\boldsymbol{M}_{n} \ddot{q}(t)+\boldsymbol{C}_{n} \dot{q}(t)+\boldsymbol{K}_{n} q(t)=\boldsymbol{\Gamma}_{n} f(t) \\
\boldsymbol{M}_{n}=\boldsymbol{I} \\
\boldsymbol{C}_{n}=2 \operatorname{diag}\left[\begin{array}{llll}
\omega_{1} \zeta_{1} & \omega_{2} \zeta_{2} & \cdots & \omega_{n} \zeta_{n}
\end{array}\right] \\
\boldsymbol{K}_{n}=\operatorname{diag}\left[\begin{array}{llll}
\omega_{1}^{2} & \omega_{2}^{2} & \cdots & \omega_{n}^{2}
\end{array}\right] \\
\boldsymbol{\Gamma}_{n}=\left[\begin{array}{llll}
Y_{1}\left(x_{f}\right) & Y_{1}\left(x_{f}\right) & \cdots & Y_{1}\left(x_{f}\right)
\end{array}\right]
\end{gathered}
$$

式中, $\boldsymbol{M}_{n}$ 为质量矩阵, $\boldsymbol{C}_{n}$ 为阻尼矩阵, $\boldsymbol{K}_{n}$ 为刚度 矩阵, $\boldsymbol{\Gamma}_{n} f(t)$ 为外激励矢量。

\section{2 控制方案设计}

基于上述理论分析, 建立如图 6 所示的系统控 制方案。利用 ADAMS 与 Matlab 软件联合仿真, 建 立索并联抑振机构的仿真模型, 并在 Simulink 环境 中搭建整个系统的控制模型，如图 7 所示。以柔性 桁架与绳索的铰接点作为位置与速度的采样点, 将 与平衡位置的偏差作为模糊 PID 控制器的输入, 得 到的 4 索索力值作为柔性桁架模型的外加激励力以 抑制其振动。图 7 中的 admas_sub 为柔性桁架仿真 模型, 模糊控制器的两个输入通道分别为采样点的 位置偏差与速度偏差，输入 1 对应的 3 个三角形隶 属函数 DYFU, DYZERO 与 DYZHENF 如图 8 所示, 输入 2 对应的 3 个三角形隶属函数 VYFU, VYZERO 与 VYZHENF 如图 9 所示, 模糊控制规则如表 1 所 示，对应的模糊规则曲面图如图 10 所示，模糊控制 器的 9 个输出三角形隶属函数 $\mathrm{n} 11 \sim \mathrm{n} 19$ 如图 11 所 示。柔性桁架仿真模型 admas sub 的索力 1 是初始 扰动力, 索力 2 为图 3 受力图中 $A_{3} B_{3}$ 斜拉索索力,

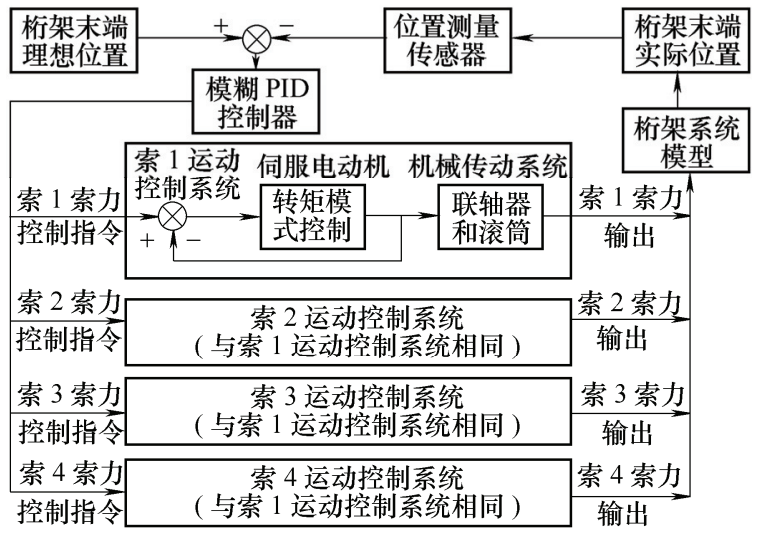

图 6 控制方案图

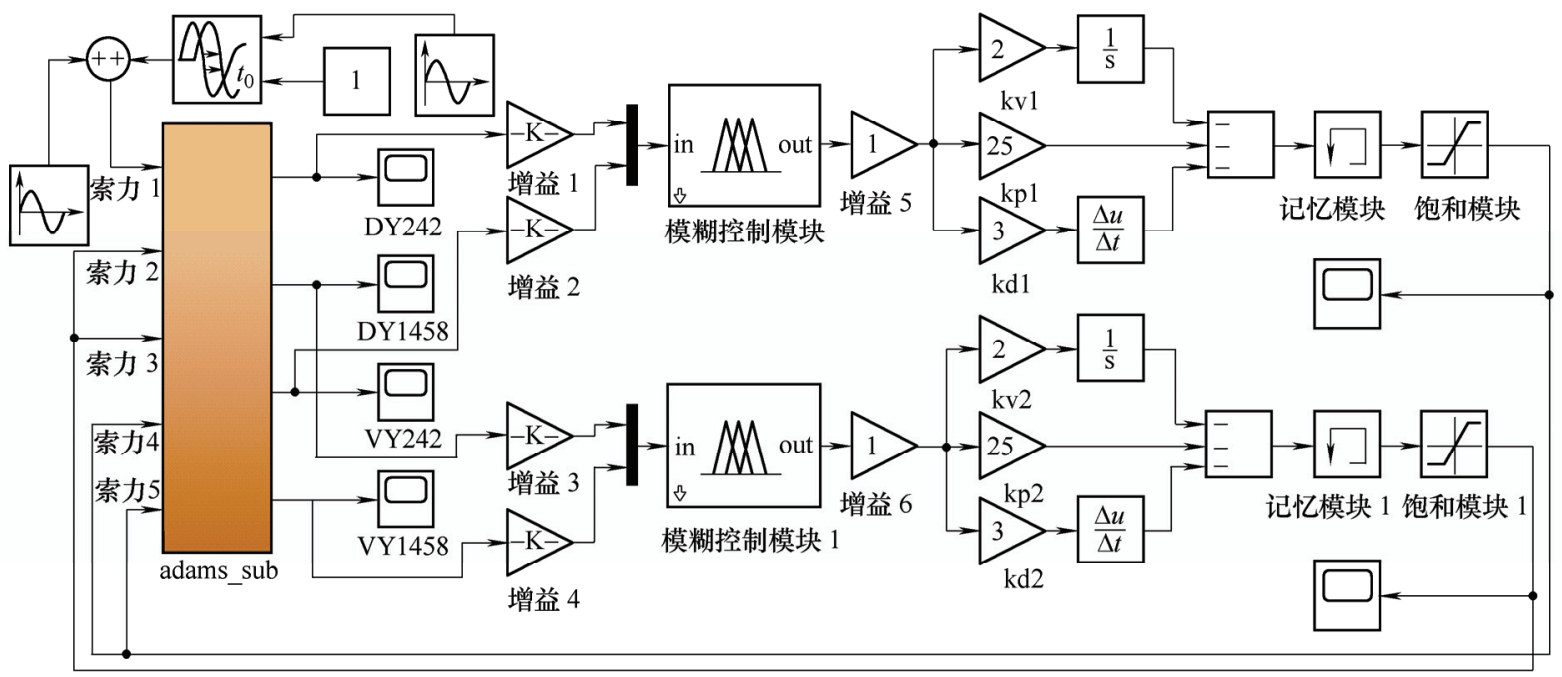

图 7 系统控制框图 


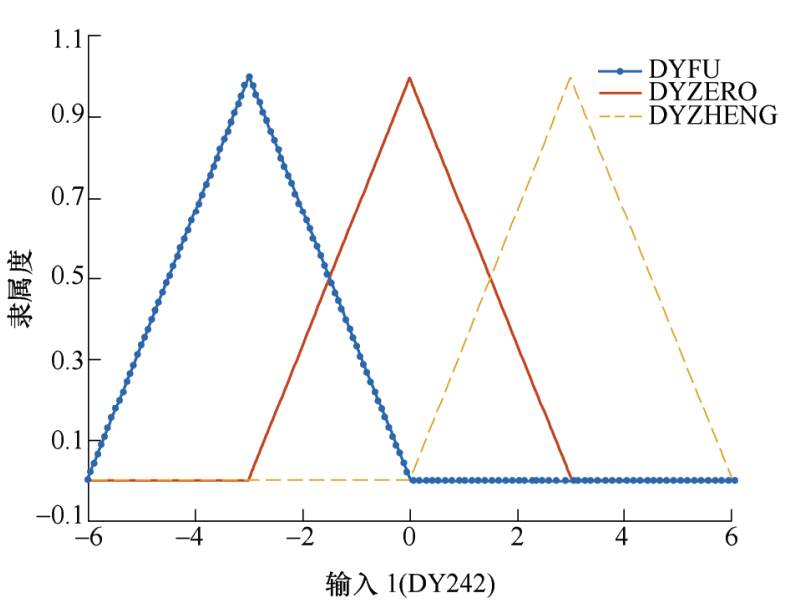

图 8 输入 1 隶属函数

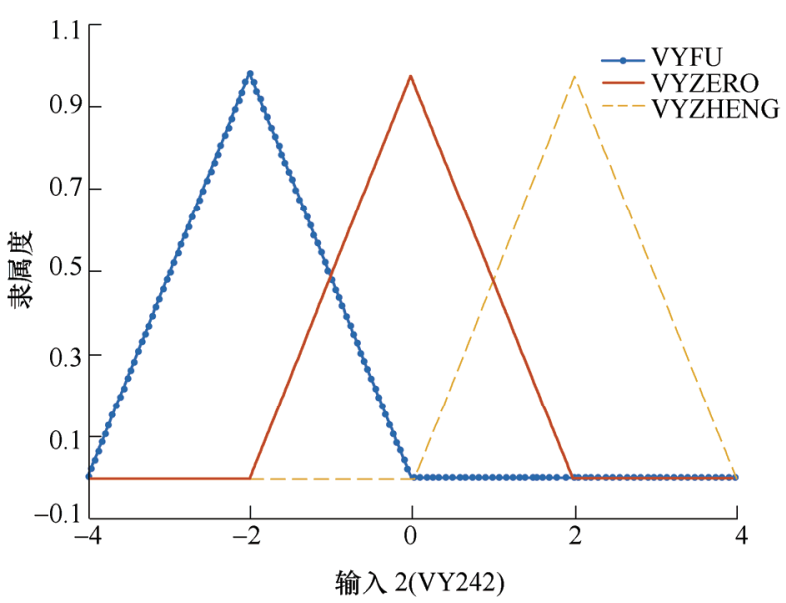

图 9 输入 2 隶属函数

表 1 模糊控制规则表

\begin{tabular}{ll}
\hline \multicolumn{1}{c}{ 输入规则 } & 输出规则 \\
\hline If(DY242 is DYZHENF) and (VY242 is VYZHENG) & PIDinput is n11 \\
If(DY242 is DYZHENF) and (VY242 is VYZERO) & PIDinput is n12 \\
If(DY242 is DYZHENF) and (VY242 is VYFU) & PIDinput is n13 \\
If(DY242 is DYZERO) and (VY242 is VYZHENG) & PIDinput is n14 \\
If(DY242 is DYZERO) and (VY242 is VYZERO) & PIDinput is n15 \\
If(DY242 is DYZERO) and (VY242 is VYFU) & PIDinput is n16 \\
If(DY242 is DYFU) and (VY242 is VYZHENG) & PIDinput is n17 \\
If(DY242 is DYFU) and (VY242 is VYZERO) & PIDinput is n18 \\
If(DY242 is DYFU) and (VY242 is VYFU) & PIDinput is n19 \\
\hline
\end{tabular}

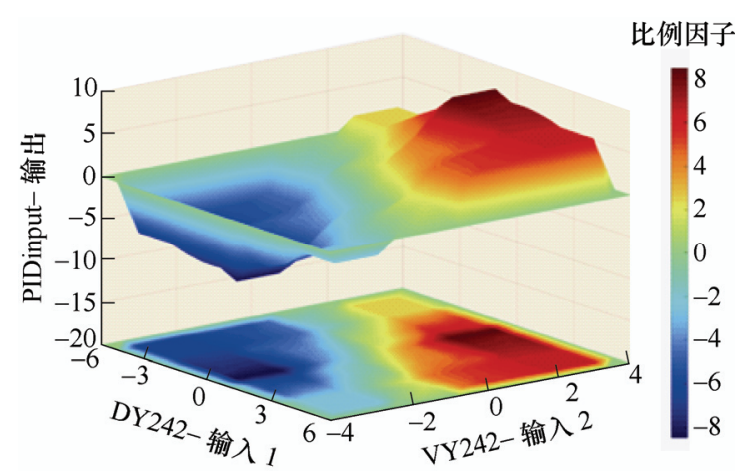

图 10 模糊规则曲面图

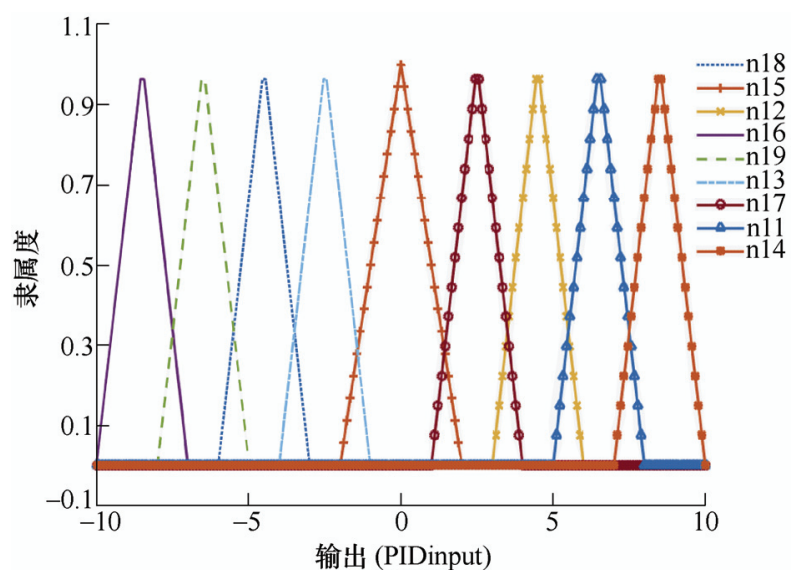

图 11 输出隶属函数

索力 3 为 $A_{2} B_{2}$ 斜拉索索力, 索力 4 为 $A_{4} B_{4}$ 斜拉索索 力, 索力 5 为 $A_{1} B_{1}$ 斜拉索索力, 输出为两个采样点 的位置偏差与速度偏差。

\section{3 控制系统仿真分析}

桁架物理模型如图 3 所示, 其结构参数如表 2 所示。

表 2 桁架结构参数

\begin{tabular}{ccc}
\hline 结构参数 & 主杆 & 副杆 \\
\hline 直径 $/ \mathrm{mm}$ & 160 & 84 \\
壁厚 $/ \mathrm{mm}$ & 0.3 & 2 \\
长度 $/ \mathrm{m}$ & 60 & $6 / 3.6$ \\
弹性模量 $/ \mathrm{GPa}$ & 8 & 11000 \\
密度 $/\left(\mathrm{kg} / \mathrm{m}^{3}\right)$ & 1630 & 1900 \\
\hline
\end{tabular}

依据表 2 列出的柔性桁架结构参数, 建立上述 桁架物理模型的仿真模型 admas_sub。初始零时刻 在桁架自由端施加一脉冲激励力, 使得采样点 3 的 初始位置偏离平衡位置 $530 \mathrm{~mm}$, 仿真得到采样点 3 自由状态下的振幅与速度的激励响应曲线, 如图 12、13 所示, 以采样点振幅达到 $\pm 20 \mathrm{~mm}$ 内视为桁 架结构停振, 则自然状态下需要 $937 \mathrm{~s}$ 。对振幅曲线 进行快速傅里叶 3D 变换, 如图 14 所示, 可得柔性 桁架结构的第一阶固有频率为 $0.056 \mathrm{~Hz}$, 与 ANSYS 计算结果相符。

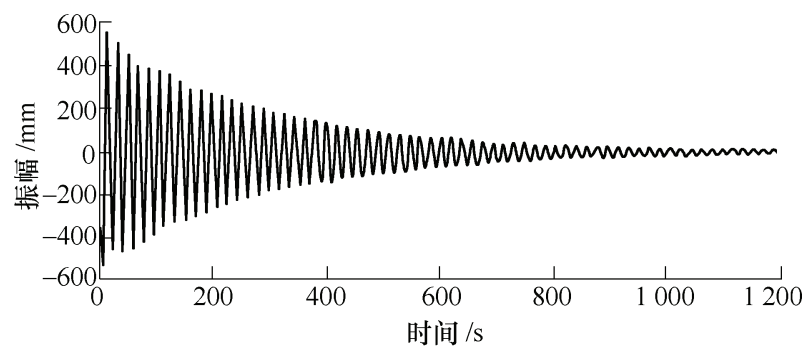

图 12 采样点 3 自由状态振幅曲线 


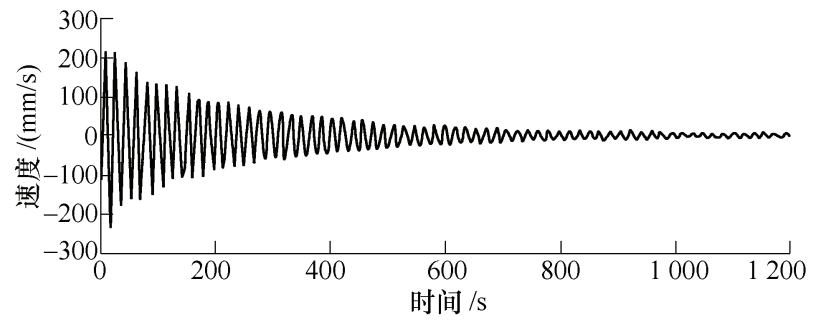

图 13 采样点 3 自由状态速度曲线

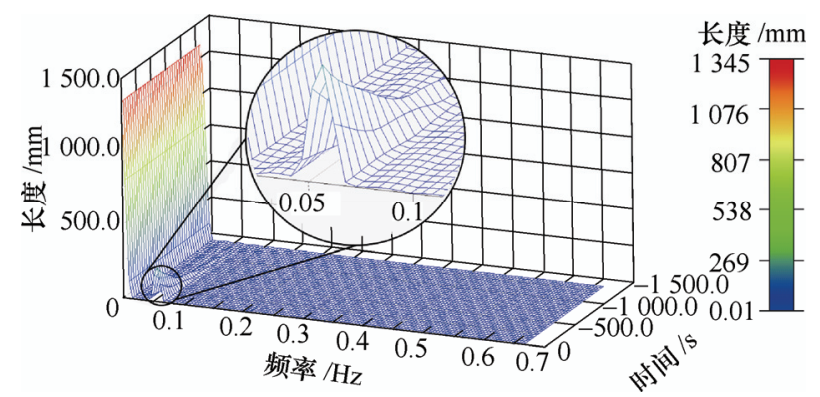

图 14 快速傅里叶变换图像

将采样点 3 与采样点 30 作为柔性桁架结构与绳 索的铰接点, 4 根斜拉索布局形式如图 2 所示。建 立 4 索桁架仿真模型, 并利用上一节构建的控制系 统进行仿真分析。同样, 初始零时刻在析架自由端 施加一脉冲激励力, 使得采样点 3 的初始位置偏离 平衡位置 $530 \mathrm{~mm}$ 。为了对比绳索抑振的效果, 将设 置两组控制参数分别进行仿真, 两组控制参数设置 如表 3 所示, 各个参数在系统控制框图中的作用如 图 7 所示。

表 3 控制参数表

\begin{tabular}{ccc}
\hline 控制参数 & 第一组 & 第二组 \\
\hline Gain6/ Gain7 & 1 & 5 \\
Gain9/ Gain11 & 0.01 & 0.01 \\
Gain8/ Gain10 & 0.01 & 0.01 \\
kv1 & 4 & 0 \\
kp1 & 25 & 5 \\
kd1 & 4 & 0 \\
kv2 & 0 & 0 \\
kp2 & 25 & 5 \\
kd2 & 1 & 0 \\
\hline
\end{tabular}

采用第一组控制参数进行仿真得到采样点 3 的 振幅与速度曲线如图 15、16 所示, 图 3 受力图中 $A_{2} B_{2}$ 与 $A_{3} B_{3}$ 两根斜拉索的索力如图 17 所示, $A_{1} B_{1}$ 与 $A_{4} B_{4}$ 两根斜拉索的索力如图 18 所示。对比图 15 与 12 可知, 使用控制策略后, 采样点的振幅变化范 围由 $530 \mathrm{~mm}$ 减小为 $400 \mathrm{~mm}, a$ 点为振幅达到 \pm 20 $\mathrm{mm}$ 的第一点, 衰减时间减小为 $166 \mathrm{~s}$ 。

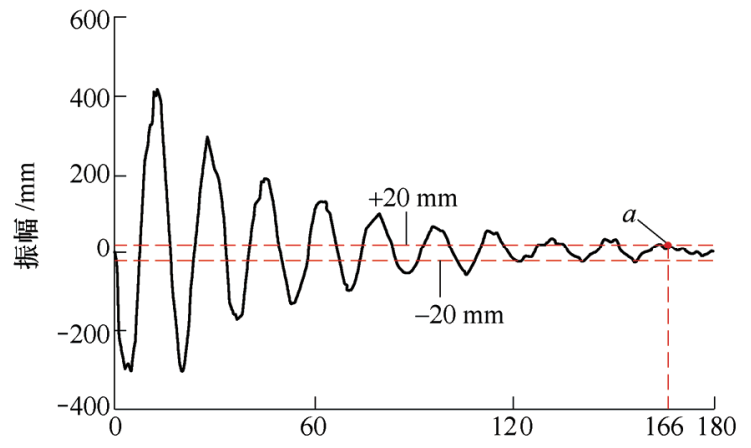

时间 $/ \mathrm{s}$

图 15 采样点 3 强迫响应振幅曲线

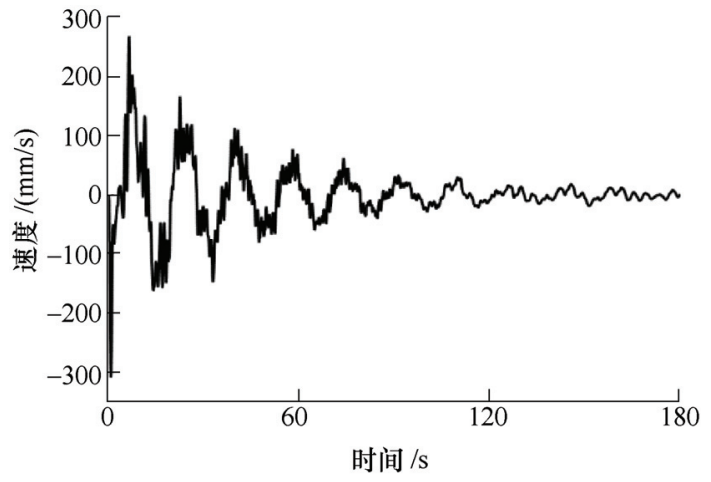

图 16 采样点 3 强迫响应速度曲线

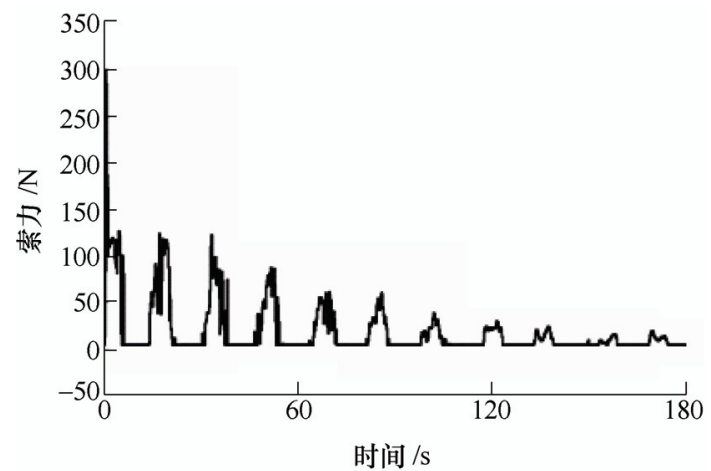

图 $17 A_{2} B_{2}$ 与 $A_{3} B_{3}$ 斜拉索索力

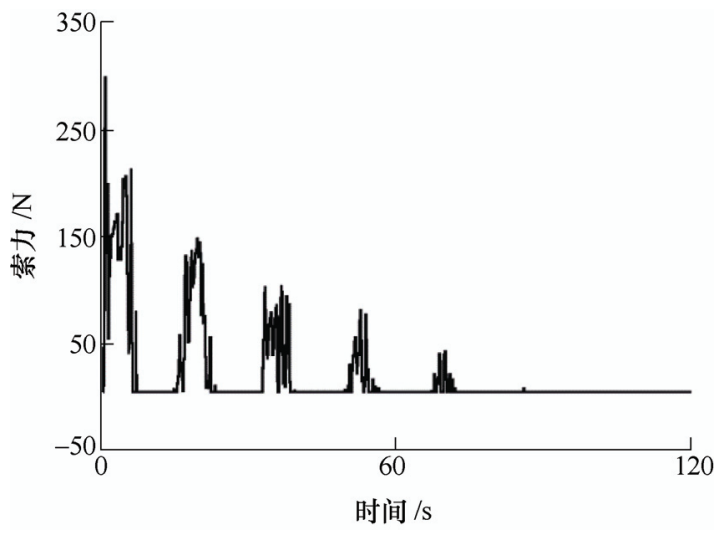

图 $18 A_{1} B_{1}$ 与 $A_{4} B_{4}$ 斜拉索索力

由图 17、18 可知, 4 根斜拉索索力变化范围为 5 300 N，保证了绳索无虚牵，且其幅值是间歇变 化的, 这与绳索只能承受拉力而无法承受压力以及 桁架周期性的往复振动有关。伺服电动机扭矩及功 
率选择的与索力大小有直接关系, 考虑到卫星体积 与重量的限制及柔性桁架可承受力, 限制索力的大 小是必要的。

因此, 为进一步缩短衰减时间, 减小斜拉索索 力, 对控制参数进行优化, 采用第二组控制参数进 行仿真得到采样点 3 的振幅与速度曲线如图 19、20 所示, 图 3 受力图中 $A_{2} B_{2}$ 与 $A_{3} B_{3}$ 两根斜拉索的索力 如图 21 所示, $A_{1} B_{1}$ 与 $A_{4} B_{4}$ 两根斜拉索的索力如图 22 所示。

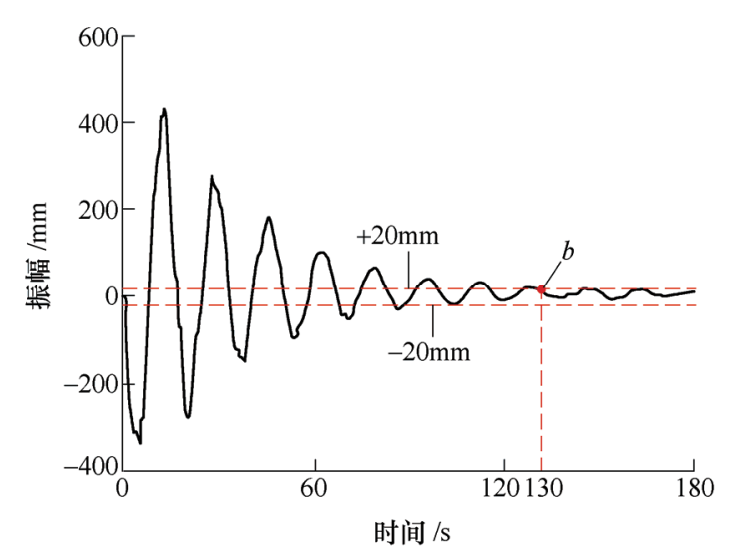

图 19 采样点 3 强迫响应振幅曲线

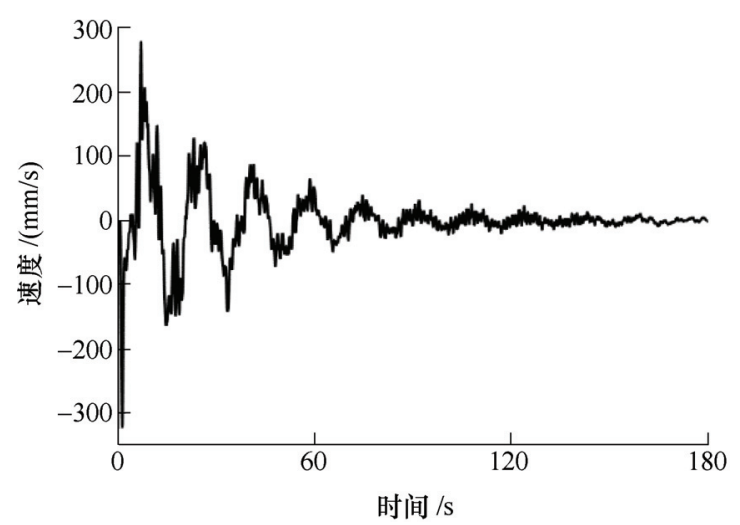

图 20 采样点 3 强迫响应速度曲线

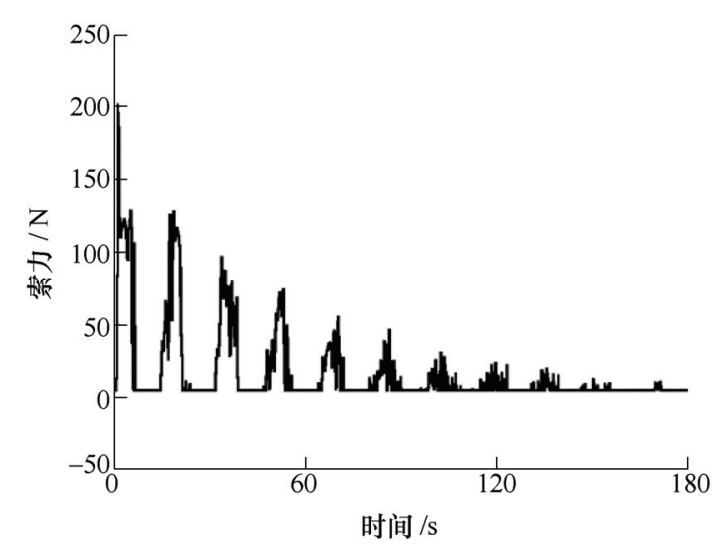

图 $21 A_{2} B_{2}$ 与 $A_{3} B_{3}$ 斜拉索索力

图 19 中 $b$ 点为振幅达到 $\pm 20 \mathrm{~mm}$ 的第一点, 与 图 15 对比可知, 采用第二组控制参数后, 振动衰减 时间缩短为 $130 \mathrm{~s}$, 比 $a$ 点的衰减时间缩短了 $36 \mathrm{~s}$,

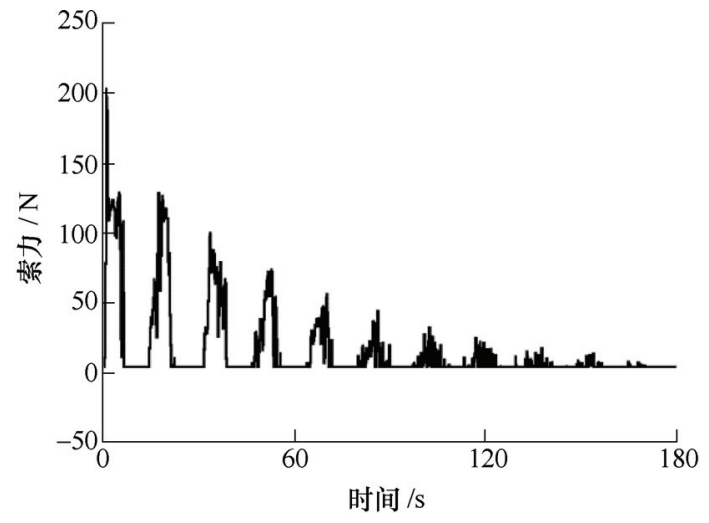

图 $22 A_{1} B_{1}$ 与 $A_{4} B_{4}$ 斜拉索索力

仅为控制状态下衰减时间的 $13.9 \%$ 。4 根斜拉索索 力变化范围为 5 200 N, 比第一组索力最大值减小 了 $100 \mathrm{~N}$, 大大降低了对伺服电动机扭矩与功率的 要求, 这对于减小卫星自身体积与重量是至关重要 的。根据全文分析, 可给出最终模糊 PID 控制参数 如表 3 第二组所示。

\section{4 结论}

本文提出了一种四索驱动的柔性抑振并联机 构, 用于超长尺度柔性桁架结构的振动主动抑制, 并根据闭环矢量原理和机构的几何特征, 利用振型 叠加法分析了外部激励作用下的系统强迫响应特 性, 仿真结果表明, 借助模糊 PID 控制策略, 能够 极大缩短桁架结构的振动衰减时间和减小索力变化 范围上限值, 证明了采用索机构进行超长柔性桁架 结构的主动抑振控制可行性问题。本文的研究将为 基于柔索并联机构的振动抑制应用奠定理论基础。

\section{参 考 文 献}

[1] 楚中毅, 马静, 李丹. 空间柔性伸杆机构振动抑制的半 物理仿真试验 $[\mathrm{J}]$. 机械工程学报, 2012, 48(21): 8-14. CHU Zhongyi, MA Jing, LI Dan. Semi-physical simulation experiments on vibration control of flexible space manipulator[J]. Journal of Mechanical Engineering, 2012, 48(21): 8-14.

[2] XIAO B, HU Q, ZHANG Y. Adaptive sliding mode fault tolerant attitude tracking control for flexible spacecraft under actuator saturation[J]. IEEE Transactions on Control Systems Technology, 2012, 20(6): 1605-1612.

[3] MALEKZADEH M, KARIMPOUR H. Adaptive super twisting vibration control of a flexible spacecraft with state rate estimation[J]. Journal of Sound and Vibration, 2018, 422: 300-317.

[4] JAMSHIDIFAR H, KHOSRAVANI S, FIDAN B, et al. Vibration decoupled modeling and robust control of 
redundant cable-driven parallel robots[J]. IEEE/ASME Transactions on Mechatronics, 2018, 23(2): 690-701.

[5] JI N, LIU J. Vibration control for a flexible satellite with input constraint based on Nussbaum function via back stepping method[J]. Aerospace Science \& Technology, 2018, 77: 563-572.

[6] 陶佳伟, 张涛. 控制受限的挠性航天器姿态机动控制和 振动抑制[J]. 电机与控制学报，2018(3): 105-113.

TAO Jiawei, ZHANG Tao. Robust adaptive attitude maneuvering and vibration reducing control of flexible spacecraft with input saturation[J]. Electric Machines and Control, 2018(3): 105-113.

[7] 许建国, 邵康, 张静静. 空间析架结构的优化配置及振 动控制研究 [J]. 计算机测量与控制, 2016, 24(3): 67-70. XU Jianguo, SHAO Kang, ZHANG Jingjing. Research on optimal allocation and vibration control of space truss structure[J]. Computer Measurement \& Control, 2016, 24(3): $67-70$.

[8] 张金龙, 卢少波, 时军委. 驱动激励下的柔性太阳翼系 统振动及抑制研究[J]. 载人航天, 2017, 23(4): 487-492. ZHANG Jinlong, LU Shaobo, SHI Junwei. Research on vibration and its attenuation of flexible solar array induced by sun-tracking driving[J]. Manned Spaceflight, 2017, 23(4): 487-492.

[9] 姚荵, 唐晓强, 汪劲松. 射电望远镜馈源支撑系统索力 特性研究[J]. 自然科学进展, 2009, 19(11): 1221-1229. YAO Rui, TANG Xiaoqiang, WANG Jinsong. Cable tension analysis and optimization of radio telescope feed support system[J]. Progress in Natural Science, 2009, 19(11): 1221-1229.

[10] LAPIDUS S G. The factors to be considered in the correct cable for variable frequency drive applications on cranes[C]// Industry Applications Conference, 1998. Thirty-Third IAS Annual Meeting. IEEE, 1998: 2257-2262.
[11] CONG B P, SONG H Y, YANG G. Tension analysis of cable-driven parallel mechanisms $[\mathrm{C}] / /$ International Conference on Intelligent Robots and Systems, IEEE, 2005: 257-262.

[12] HILLER M, FANG S, MIELCZAREK S, et al. Design, analysis and realization of tendon-based parallel manipulators[J]. Mechanism \& Machine Theory, 2005, 40(4): 429-445.

[13] TANG X, YAO R. Dimensional design on the six-cable driven parallel manipulator of FAST[J]. Journal of Mechanical Design, 2011, 133(11): 111012.

[14] 唐乐为, 唐晓强, 汪劲松, 等. 七索并联对接机构作业 空间分析及索力优化设计 $[\mathrm{J}]$. 机械工程学报，2012， 48(21): 1-7.

TANG Lewei, TANG Xiaoqiang, WANG Jinsong, et al. Workspace analysis and tension optimization design in docking parallel mechanism driven by seven cables[J]. Journal of Mechanical Engineering, 2012, 48(21): 1-7.

[15] 张义民. 机械振动学基础[M]. 北京: 清华大学出版社, 2007.

ZHANG Yimin. Mechanical vibration[M]. Beijing : Tsinghua University Press, 2007.

作者简介: 孙海宁, 男, 1994 年出生, 博士研究生。主要研究方向为索 驱动并联机器人振动特性。

E-mail: sunhn17@mails.tsinghua.edu.cn

唐晓强(通信作者), 男, 1973 年出生, 博士, 教授, 博士研究生导师。 主要研究方向为并联机器人。

E-mail: tang-xq@mail.tsinghua.edu.cn

王晓宇, 女, 1981 年出生, 博士, 高级工程师。主要研究方向航天器结 构设计与分析, 大型空间结构动力学与控制。

E-mail: antlb@163.com

崔志伟, 男, 1989 年出生, 博士研究生。主要研究方向为索驱动并联机 器人刚度特性。

E-mail: czw16@mails.tsinghua.edu.cn

侯森浩, 男, 1992 年出生, 博士研究生。主要研究方向为索驱动并联机 器人力控特性。

E-mail: housh16@mails.tsinghua.edu.cn 